\title{
Accuracy and dosimetric parameters comparison of 3D-printed non-coplanar template-assisted computed tomography-guided iodine-125 seed ablative brachytherapy in pelvic lateral recurrence of gynecological carcinomas
}

\author{
Ang Qu, MD, Ping Jiang, MD, Shuhua Wei, PhD, Yuliang Jiang, MD, Zhe Ji, MD, Haitao Sun, MM, Weiyan Li, MB, \\ Yuxia Shao, MB, Jinghong Fan, NB, Junjie Wang, MD, PhD \\ Department of Radiation Oncology, Peking University $3^{\text {rd }}$ Hospital, Beijing, China
}

\begin{abstract}
Purpose: To investigate the accuracy of needle distribution and dosimetric parameter differences of 3D-printing non-coplanar template (3D-PNCT)-assisted computed tomography (CT)-guided iodine- 125 seed ablative brachytherapy $\left({ }^{125} \mathrm{I}-\mathrm{SAB}\right)$ in gynecological cancer patients with non-central pelvic recurrence between pre-operative plan and post-operative plan.

Material and methods: Thirty-eight patients with forty-one non-central pelvic recurrent gynecological carcinomas after radiotherapy were enrolled in this study. All patients received 3D-PNCT-assisted CT-guided ${ }^{125}$ I-SAB from January 2016 to January 2019. The position, angle, and depth of seed needles were measured in both pre-operative plan and intra-operative real-time plan in brachytherapy treatment planning system (B-TPS). Dosimetric parameters of $\mathrm{D}_{90}$ $\mathrm{D}_{100}, \mathrm{~V}_{100}, \mathrm{~V}_{150}$, and $\mathrm{V}_{200}$ as well as quality parameters of conformal index (CI), external index (EI), and homogeneity index (HI) were compared between pre-operative plan and post-operative plan. Peri-operation complications and radiation-related toxicity were assessed.

Results: Median follow-up time was 12 months (range, 5-34 months). Prescribed dose was 100-170 Gy (median, 120 Gy). Radioactivity of ${ }^{125} \mathrm{I}$ seed was $0.4-0.7 \mathrm{mCi}$ (median, $0.55 \mathrm{mCi}$ ). Mean depth deviation for 499 needles was $0.8 \pm 1.0 \mathrm{~cm}$. Mean angular deviation was $2.2 \pm 2.1$ degrees. Mean tip distance deviation of needles was $0.4 \pm 0.3 \mathrm{~cm}$. There were significant differences between pre-operative and post-operative plans in CI $(p=0.001)$ and EI $(p=0.005)$. No significant differences were shown in $\mathrm{D}_{90}, \mathrm{D}_{100}, \mathrm{~V}_{100}, \mathrm{~V}_{150}, \mathrm{~V}_{200}$, and $\mathrm{HI}$ between pre-operative and post-operative plans. Only few patients suffered from $\leq$ grade 2 toxicities.

Conclusions: 3D-PNCT-assisted CT-guided ${ }^{125} \mathrm{I}-\mathrm{SAB}$ is safe and feasible for non-central pelvic recurrence of gynecological cancer. All complications are tolerable and mild.

J Contemp Brachytherapy 2021; 13, 1: 39-45 DOI: https://doi.org/10.5114/jcb.2021.103585
\end{abstract}

Key words: interstitial permanent brachytherapy, gynecological carcinoma, 3D printing template, iodine-125.

\begin{abstract}
Purpose
High rates of morbidity and mortality associated with gynecological cancer concern all females in the world [1], with cervical cancer as the most common genital carcinoma in women. Concurrent chemoradiotherapy is the standard treatment for patients with locally advanced cervical carcinomas. According to the International Federation of Gynecology and Obstetrics (FIGO), the relapse rate of cervical cancer ranges from $11 \%$ to $22 \%$ stage IB-IIA
\end{abstract}

and $28 \%$ to $64 \%$ in FIGO stage IIB-IVA [2]. Pelvic recurrences of gynecological cancer are classified into central and non-central types. The prognosis in non-central pelvic recurrent gynecological cancer patients is very poor, with a 5 -year overall survival below $10 \%$. Also, no acceptable curative optional modality is presently available $[3,4,5,6]$. Pelvic exenteration is considered for selected patients with central recurrence, but high-risk complications are unavoidable [7,8]. Although re-irradiation is an alternative option, it is hard to deliver enough doses 
to lesions previously treated with radiotherapy $[9,10]$. In addition, chemotherapy is mostly applied as palliative treatment $[11,12]$.

Iodine-125 seed ablative brachytherapy $\left({ }^{125} \mathrm{I}-\mathrm{SAB}\right)$ is a minimally invasive modality, which allows high-dose delivery to the target and low-dose distribution to surrounding normal tissues $[13,14]$. Computed tomography (CT)-guided ${ }^{125} \mathrm{I}$-SAB is successfully applied as re-irradiation in tumors located at different sites, such as head and neck, thorax, abdomen, retroperitoneum, and vertebra $[15,16,17,18]$. Indications for ${ }^{125}$ I-SAB are being rapidly expanded, especially for recurrent solid carcinomas after external beam radiotherapy (EBRT). However, the deviation of needles puncture under CT-guidance is inevitable, and it is very difficult to keep the conformity of target doses as pre-operative plan designed pattern, since it involves the experience of operator, interference of organs at risk, and organ movements.

3D-printing non-co-planar template (3D-PNCT) was invented and integrated into CT guidance for ${ }^{125} \mathrm{I}-\mathrm{SAB}$ in our institution. The safety, accuracy, and feasibility of 3D-PNCT application in various tumors and locations has been explored $[19,20,21,22]$. In this retrospective study, we analyzed the accuracy of needle position between pre-operative plan and intra-operative plan as well as the dosimetric parameters of 3D-PNCT-assisted CT-guidance ${ }^{125} \mathrm{I}-\mathrm{SAB}$ between pre-operative plan, intraoperative plan, and post-operative plan in gynecological cancer patients with a non-central pelvic recurrence.

\section{Material and methods}

\section{Indications for selection and ${ }^{125}$ I-SAB preparation}

Thirty-eight patients with non-central pelvic recurrent cervical carcinomas treated with ${ }^{125} \mathrm{I}-\mathrm{SAB}$ between January 2016 and January 2019 were enrolled into the study (Table 1). Inclusion criteria were as follows: 1. Karnofsky performance status (KPS) score $\geq 70$ and expected survival $\geq 3$ months; 2 . Pathologically or radiologically confirmed diagnosis; 3 . Diameter of the tumor $\leq 5 \mathrm{~cm}$, recurrent location in lateral region of the pelvic;

Table 1. General information $(n=38)$

\begin{tabular}{|c|c|c|}
\hline & Numbers & $\begin{array}{c}\text { Percentage } \\
(\%)\end{array}$ \\
\hline Age (years), range (median) & $33-72(49)$ & \\
\hline \multicolumn{3}{|l|}{ Primary tumor } \\
\hline Cervical cancer & 34 & 89.5 \\
\hline Endometrial cancer & 4 & 10.5 \\
\hline \multicolumn{3}{|l|}{ Pathology } \\
\hline Squamous cell carcinoma & 31 & 81.5 \\
\hline Adenocarcinoma & 7 & 18.4 \\
\hline \multicolumn{3}{|l|}{ Previous therapy } \\
\hline Surgery & 24 & 63.2 \\
\hline Chemotherapy & 35 & 92.1 \\
\hline Radiotherapy & 38 & 100.0 \\
\hline Median dose (Gy) & $50(42-90)$ & \\
\hline
\end{tabular}

4. The number of metastasis $\leq$ three; 5 . Non-candidate for surgery or unresectable tumor, or refused to EBRT. Exclusion criteria involved: 1 . Severe organ dysfunction; 2. Coagulation dysfunctions; 3 . Recent use of anticoagulant and infection; 4. Mental illness; 5. Tumor-invaded vagina, rectum, or bladder. The study protocol was approved by the Ethics Committee of Peking University Third Hospital (BMU2017JC001-3). All participated patients provided a written informed consent.

The selected patients were immobilized with vacuum pad in supine position or prone position, and underwent $\mathrm{CT}$ simulation. Both native and contrast CT scan was performed with $5 \mathrm{~mm}$ slice thickness. Co-ordinate positioning lines were marked on the skin of patient. CT images were transferred into a brachytherapy treatment planning system (B-TPS, brachytherapy treatment planning system provided by the Beijing University of Aeronautics and Astronautics, and Beijing Astro Technology Co., Ltd., China). Both target and OARs were defined and delineated. The prescribed dose and activity of ${ }^{125}$ I seeds were determined. The implantation needle path and distribution of ${ }^{125} \mathrm{I}$ seeds were set and calculated. Doses received by $90 \%$ of gross tumor volume (GTV) $\left(\right.$ GTV $\left.D_{90}\right)$ were supposed to be as close to the prescription doses (PD) as possible, whereas doses received by organs at risk (OARs) were kept as low as possible. The median PD was $120 \mathrm{~Gy}$ (range, 110-170 Gy), the activity of ${ }^{125} \mathrm{I}$ seeds ( ${ }^{125} \mathrm{I}$ seed model 6711-99; length $4.5 \mathrm{~mm}$, diameter $0.8 \mathrm{~mm}$, half-life 59.4 days; Beijing Atom High Tech Pharmaceutical Company Inc., China) were $0.4-0.7 \mathrm{mCi}$, with median $0.55 \mathrm{mCi}$ $(1 \mathrm{Ci}=3.7 \times 1010 \mathrm{~Bq})$.

\section{D-PNCT design and production}

The seed needle distribution in the target was designed in B-TPS pre-operative plan, and kept in parallel way if possible. In the meanwhile, OARs, such as blood vessels, bone, and nerves, were avoided or kept away from needles pathways, with a safety distance of $0.5-1.0 \mathrm{~cm}$.

The B-TPS datasets were transferred into Magics 19.01 (Materialise, Belgium) for digital modeling of 3D individualized template. Information about the needle pathway was added to the model. The individual template was printed on a 3D light-cured rapid forming printer (RS6000, Shanghai Liantaiv 3D Technology Company Inc., China). The individual template included body surface morphology of treated area, X-axis, and Y-axis coordinate registered marks and the simulated needle path information. Two or three fixation needles were used to make sure that the 3D-PNCT was aligned with patient body (Figure 1).

\section{Workflow of ${ }^{125} \mathrm{I}-\mathrm{SAB}$}

The ${ }^{125}$ I-SAB procedure was as follows: 1 . Spinal anesthesia was carried out. Patients were immobilized with vacuum pad on a setup position. The 3D-PNCT was aligned to the patient body by $\mathrm{X}$-axis and $\mathrm{Y}$-axis positioning reference lines. Two or three fixation needles were inserted into the patient's body, $2-3 \mathrm{~cm}$ in depth; 2 . CT scan was performed to determine the accurate position of 

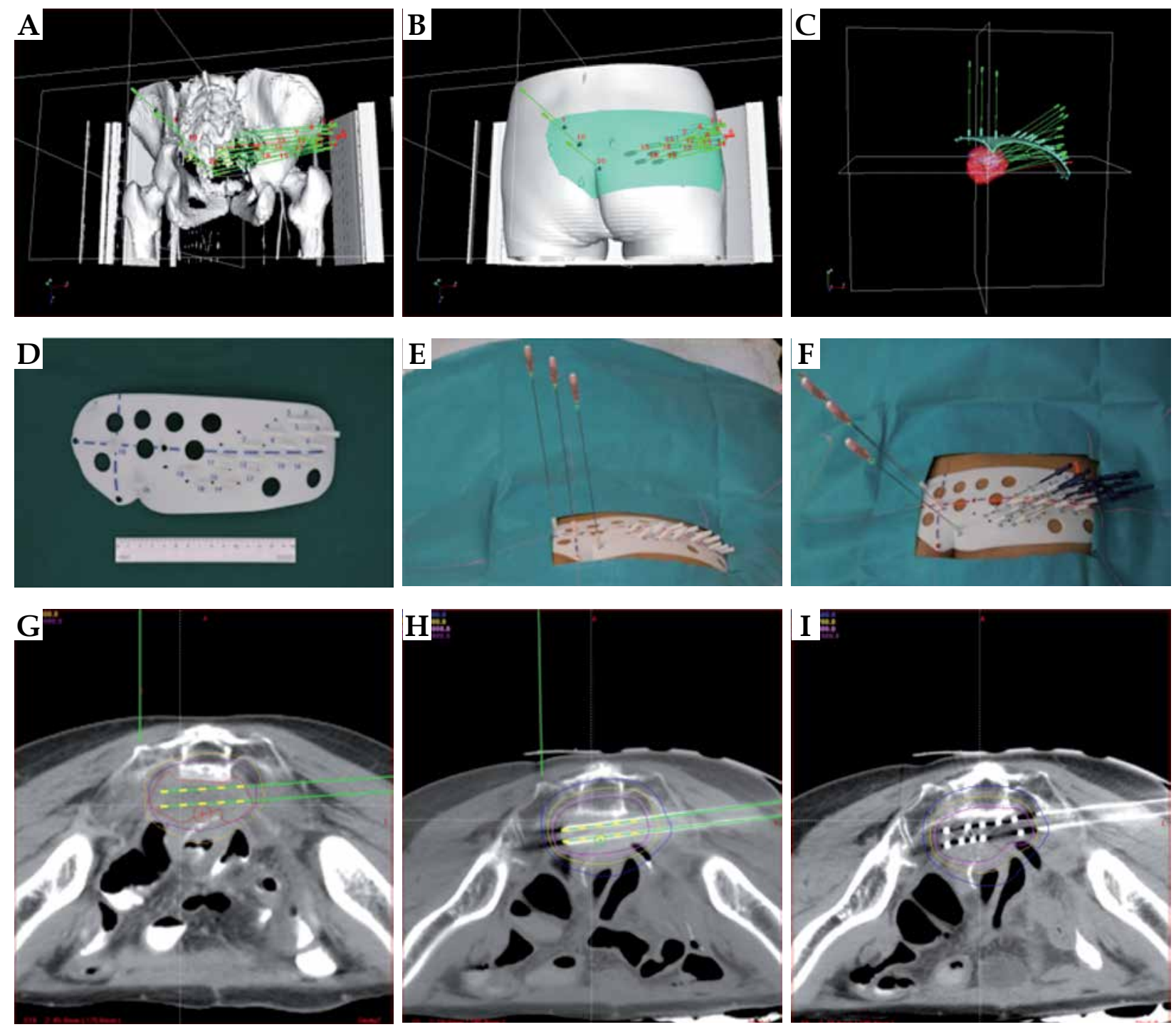

Fig. 1. Procedure of the 3D-PNCT-assisted CT guidance ${ }^{125}$ I SAB. A) Simulation of needles and template distribution based on pre-operative CT images, B) 3D reconstruction of needles and 3D-PNCT, C) 3D reconstruction of needles, D) 3D-PNCT, E) stable needles insertion, F) seed needles insertion, G) CT image of pre-operative planning, H) intra-operative needles distribution, I) seed distribution into the target

fixation needles according to the designed pre-operative plan; 3. If the fixation needles position was mismatched between real-time images and pre-operative plan images with a deviation of more than $2 \mathrm{~mm}$, it was finely adjusted; 4 . The needles were all inserted into the targets; 5. CT scan was performed again to confirm the position of needle tips, if the deviation was more than $2 \mathrm{~mm}$, a fine adjustment was made; 6 . Another CT scan was performed to verify the position of the implantation needle compared with pre-operative plan, and intra-operation real-time doses optimization was performed; $7 .{ }^{125} \mathrm{I}$ seed implantation was delivered with an applicator (Mick 200-TPV, Mick Radio-Nuclear Inc., USA); 8. CT scan was performed again to confirm the actual distribution of ${ }^{125}$ I seeds within the targets. All CT images were transferred into the B-TPS for doses evaluation of post-operative plan. Patients would be discharged 1-2 days after ${ }^{125} \mathrm{I}-\mathrm{SAB}$ procedures.

\section{Post-operative plan doses evaluation}

The dose parameters comparison included the dose delivered to $90 \%\left(\mathrm{D}_{90}\right)$ and $100 \%\left(\mathrm{D}_{100}\right)$ of GTV, and volume percentage of GTV receiving $100 \%\left(\mathrm{~V}_{100}\right), 150 \%$ $\left(\mathrm{V}_{150}\right)$, and $200 \%\left(\mathrm{~V}_{200}\right)$ of PD between pre-operative and post-operative plans. The quality of targets doses distribution was represented by external index (EI), conformal index $(\mathrm{CI})$, and homogeneity index $(\mathrm{HI})$ of targeted volumes between pre-operative plan and post-operative plan. The $\mathrm{CI}$ of doses distribution in targets was evaluated by $\mathrm{CI}[23] . \mathrm{CI}=\left(\mathrm{VT}_{\text {ref }} / \mathrm{VT}\right) \times\left(\mathrm{VT}_{\text {ref }} / \mathrm{V}_{\text {ref }}\right)$, where $\mathrm{VT}$ is the volume of GTV in $\mathrm{ml}, \mathrm{VT}_{\text {ref }}$ is the volume of GTV receiving the $P D$, and $V_{\text {ref }}$ is the total volume receiving the PD. The best CI was 1, which indicated that GTV was just covered by the PD and the dose outside GTV was lower than the PD. The volume exceeding the PD outside GTV was described by EI [24]. EI $=\left(\mathrm{V}_{\text {ref }}-\mathrm{VT}_{\text {ref }}\right) / \mathrm{VT}$. 
The greater the value of the EI, the greater the recommended dosage received outside GTV. The uniformity of dose distribution was evaluated by $\mathrm{HI}$ [24]. $\mathrm{HI}=\left(\mathrm{VT}_{\text {ref }}-\right.$ $\left.\mathrm{VT}_{1.5 \mathrm{ref}}\right) / \mathrm{VT}_{\text {ref, }}$, where $\mathrm{VT}_{1.5 \mathrm{ref}}$ was the volume of GTV, in which the dose exceeded 1.5 times of the PD. The closer to $100 \%$ of $\mathrm{HI}$, the more uniform dose distribution of GTV.

\section{Follow-up and examinations}

Follow-up assessments were performed every 3 months for the first 2 years, and every 6 months from $3^{\text {rd }}$ to $5^{\text {th }}$ year. The assessments involved routine outpatient examination and telephone interviews. The imaging examination of CT or magnetic resonance imaging (MRI) scans, even positron emission tomography (PET)-CT scans, were used to evaluate the tumor response for each follow-up visit.

The primary end-points of the study were as follows: 1. The accurate assess of needles distribution between pre-operative plan and intra-operative plan, the dosimetric parameters, such as $\mathrm{D}_{90}, \mathrm{D}_{100}, \mathrm{~V}_{100}, \mathrm{~V}_{150}$, and $\mathrm{V}_{200}$ between pre-operative and post-operative plans. The quality parameters, such as $\mathrm{CI}, \mathrm{HI}$, and EI between pre-operative and post-operative plans; 2 . The puncture-related peri-operation complications, such as bleeding, infection, fever, and pain was assessed using CTCAE 5.0 [25].

\section{Statistical analysis}

Statistical analysis was performed by SPSS version 25.0 (IBM Corp., Armonk, NY, USA). Paired sample $t$-test was applied to compare the mean of pre-operative and post-operative dosimetric parameters. $P<0.05$ was considered statistically significant.

\section{Results}

The median follow-up was 12 months (range, 5-35 months). The number of needles for pre-operative plan was 501 in 41 lesions, the actuarial number of needles for post-operative plan was 507. The number of seeds in pre-operative plan was 16-164 (median, 57), and the number of seeds in post-operative plan was 16-183 (median, 63).

The mean angular deviation was $2.20 \pm 2.10$ degrees $(p<0.001)$. The mean depth deviation for all 499 needles was $0.8 \pm 1.0 \mathrm{~cm}(p<0.001)$. The needle tip deviation was $0.4 \pm 0.3 \mathrm{~cm}$ (Table 2). The differences of $\mathrm{D}_{90}, \mathrm{D}_{100}$, $\mathrm{V}_{100}, \mathrm{~V}_{150}, \mathrm{~V}_{200}$, and $\mathrm{HI}$ between pre- and post-operative plans were not statistically significant $(p>0.05)$. There was a significantly difference between pre-operative and intra-operative plans in CI $(p=0.005)$ and between intra-operative and post-operative plans in EI, while the differences of pre- and post-operative plans of CI $(p=0.001)$ and EI were statistically significant $(p=0.005)$ (Table 3).

There was no more than grade 2 peri-operative complications, such as bleeding, infection, fever, and pain. Five patients experienced grade 1 pain after ${ }^{125} \mathrm{I}-\mathrm{SAB}$. One patient suffered from grade 1 fever, and two patients experienced grade 1 hematuria (Table 4 ).

Table 2. Deviation of needles during seeds implantation $(n=499, \bar{\chi} \pm s)$

\begin{tabular}{lcccc} 
& Pre-operative plan & Intra-operative plan & Deviation $^{*}$ & $P$-value \\
\hline Angle $\left({ }^{\circ}\right)$ & $90.1 \pm 33.5$ & $90.6 \pm 33.1$ & $2.2 \pm 2.1$ & $<0.001$ \\
\hline Depth $(\mathrm{cm})$ & $12.2 \pm 2.5$ & $12.0 \pm 2.6$ & $0.8 \pm 1.0$ & $<0.001$ \\
\hline Needle tip distance $^{*}(\mathrm{~cm})$ & - & - & $0.4 \pm 0.3$ & -
\end{tabular}

Data are expressed as $\bar{\chi} \pm s$.

Table 3. Physical characteristics of ${ }^{125}$ l seeds implantation $(\chi \pm \mathrm{s})$

\begin{tabular}{lcccccc} 
Parameter & $\begin{array}{c}\text { Pre-operative } \\
\text { plan }\end{array}$ & $\begin{array}{c}\text { Intra-operative } \\
\text { plan }\end{array}$ & $\begin{array}{c}\text { Post-operative } \\
\text { plan }\end{array}$ & $P$-value & $P$-value ${ }^{2}$ & $P_{\text {-value }}$ \\
\hline Number of needles & $13 \pm 7$ & $13 \pm 7$ & $13 \pm 7$ & 0.268 & 0.822 & 0.279 \\
\hline Number of seeds & $64 \pm 33$ & $67 \pm 32$ & $67 \pm 35$ & $<0.001$ & 0.963 & 0.005 \\
\hline GTV volume $\left(\mathrm{cm}^{3}\right)$ & $47.4 \pm 59.2$ & $47.2 \pm 59.0$ & $47.2 \pm 59.2$ & 0.776 & 0.510 & 0.951 \\
\hline $\mathrm{D}_{90}(\mathrm{~Gy})$ & $142.4 \pm 21.1$ & $142.1 \pm 23.2$ & $141.5 \pm 23.6$ & 0.843 & 0.777 & 0.656 \\
\hline $\mathrm{D}_{100}(\mathrm{~Gy})$ & $69.9 \pm 23.4$ & $71.0 \pm 25.3$ & $74.9 \pm 24.7$ & 0.798 & 0.109 & 0.147 \\
\hline $\mathrm{V}_{100}(\%)$ & $93.8 \pm 3.9$ & $93.0 \pm 6.0$ & $92.8 \pm 6.7$ & 0.187 & 0.836 & 0.185 \\
\hline $\mathrm{V}_{150}(\%)$ & $75.1 \pm 17.5$ & $71.7 \pm 13.0$ & $71.1 \pm 14.8$ & 0.241 & 0.670 & 0.167 \\
\hline $\mathrm{V}_{200}(\%)$ & $45.4 \pm 16.2$ & $46.6 \pm 16.7$ & $46.7 \pm 17.2$ & 0.606 & 0.960 & 0.572 \\
\hline $\mathrm{Cl}$ & $0.54 \pm 0.17$ & $0.51 \pm 0.18$ & $0.49 \pm 0.19$ & 0.005 & 0.126 & 0.001 \\
\hline $\mathrm{El}$ & $0.84 \pm 0.80$ & $1.03 \pm 1.04$ & $1.13 \pm 1.02$ & 0.065 & 0.014 & 0.005 \\
\hline $\mathrm{HI}$ & $0.23 \pm 0.09$ & $0.23 \pm 0.10$ & $0.29 \pm 0.29$ & 0.631 & 0.220 & 0.172
\end{tabular}

$D_{90}, D_{100}$-dose delivered to $90 \%$ or $100 \%$ of the gross tumor volume, $V_{100}, V_{150}, V_{200}$-percentage of the volume receiving $100 \%, 150 \%, 200 \%$ of the prescription dose, $\mathrm{Cl}$-conformal index, $\mathrm{HI}$ - homogeneity index, El - external index, ${ }^{1}$ pre-operative plan vs. intra-operative plan, ${ }^{2}$ intra-operative plan vs. post-operative plan, ${ }^{3}$ pre-operative plan vs. post-operative plan 


\section{Discussion}

Radiotherapy combined with chemotherapy is the main treatment for patients with unresectable gynecological cancer, most of them resulting with excellent optimal outcomes. However, there are still some patients who experience recurrences in pelvic or pelvic combined with extra-pelvic recurrence $[26,27]$. The salvage treatment for recurrent patients after EBRT is still sub-optimal. Laterally extended endopelvic resection with or without intra-operative radiotherapy may be a choice, but severe intestinal and neurological toxicities might occur in 25-30\% of patients [28]. Also, there is no significant progression for system therapy. The re-irradiation may cause severe toxicities, including intestine fistula, subcutaneous tissue fibrosis, bone necrosis, and lower limb lymphedema [4]. There is no optimum modality for patients with recurrence in non-central pelvic after EBRT [8].

With the development of ${ }^{125} \mathrm{I}-\mathrm{SAB}$, its advantage of delivering high-dose to the target while sparing the surrounding normal tissues with rapidly dose drop-off, proved ${ }^{125} \mathrm{I}-\mathrm{SAB}$ to be a unique option as a monotherapy or combined with EBRT for cancer treatment [29,30,31]. In 2015, CT guidance was integrated into ${ }^{125} \mathrm{I}-\mathrm{SAB}$, which provided a new salvage solution for recurrent carcinomas after EBRT [29,30,31,32]. However, the dosimetric patterns in post-operative plan cannot be accurately implemented compared to pre-operative plan requirements under free-hand CT guidance [33]. The multiple needles puncture accuracy was still uncertain, and procedures were unrepeatable, even for experienced personnel.

$\mathrm{Qu}$ et al. reported their results about ultrasound/CT guidance of ${ }^{125}$ I SAB for recurrent cervical cancer after previous EBRT [34], and observed no lethal toxicities. One patient had a vaginal fistula, which was considered as radiation-related injury. ${ }^{125} \mathrm{I}$ SAB could markedly release the pain. The 1- and 2-year local progression-free survival (LPFS) rates were $34.9 \%$ and $20 \%$, respectively. The 1- and 2-year overall survival (OS) rates were 52.0\% and $19.6 \%$, respectively. Recurrence site, tumor volume, and dose were the main factors affecting efficacy. The preliminary results showed that $\mathrm{D}_{90}$ ranges were wide, and that a favorable prognosis had an association with $D_{90}$ $\geq 105$ Gy. Compared to central recurrence, image-guided ${ }^{125}$ I-SAB was safe, effective, and more suitable for patients with pelvic wall recurrent cervical cancer after EBRT.

The advantages of CT-guided ${ }^{125} \mathrm{I}$-SAB include high-resolution, real-time image reconstruction, and contrast scans, which make the targets' demonstration more clearly compared with ultrasound guidance. However, there are some drawbacks of CT guidance: 1 . It takes a long time to train a skilled puncture personnel; 2 . The quality assurance of ${ }^{125} \mathrm{I}-\mathrm{SAB}$ is difficult to be kept in agreement with pre-operative plan due to OARs interferences; 3 . The procedure takes time, with an average of 2-3 hours; 4 . It poses an excessive radiation exposure to the patients. All these factors limit the use of ${ }^{125} \mathrm{I}-\mathrm{SAB}$.

$3 \mathrm{D}$ printing technique was suggested as an additive manufacturing or rapid prototyping [35]. The process first uses computer-aided design software to create a virtual $3 \mathrm{D}$ object, which is then computationally sliced into thin
Table 4. Peri-operative complication of seeds implantation (CTCAE 5.0)

\begin{tabular}{lcccc} 
Complication & Grade 1 & Grade 2 & Grade 3 & Grade 4 \\
\hline Pain & 5 & 0 & 0 & 0 \\
\hline Fever & 1 & 0 & 0 & 0 \\
\hline Hematuria & 2 & 0 & 0 & 0 \\
\hline Infection & 0 & 0 & 0 & 0
\end{tabular}

layers. The recently developed 3D printing technique has been widely applied in medical care [36,37]. Brachytherapy (BT) plays a very important role in the treatment of breast, prostate, and skin cancers as a monotherapy or combined with EBRT. Quality assurance in BT is basically dependent on template assistance. Image guidance combined with template assistance, made BT a unique modality for cancer treatment. However, the indications of BT were still limited due to template techniques and image guidance immaturity, until 3D printing template methods development. The 3D-PNCT was invented, and the software program was integrated with B-TPS. The needles position, angles, and direction can be reverse-designed to meet the target volume of doses requirements and to avoid OARs. In the meantime, 3D-PNCT obtained a co-ordinate, which was the reference for immobilization with patient's body. The precision and accuracy of ${ }^{125}$ I-SAB has been significantly improved $[38,39,40,41]$.

The results of the present study showed that 3D-PNCT combined with CT guidance could greatly reduce the misalignment error and improve the accuracy of needles puncture for non-central pelvic lesions. Under the 3D-PNCT-assisted CT guidance, each needle could be accurately placed in the target. Therefore, the exact doses delivered to the targets could meet the requirements of pre-operative plan. 3D-PNCT-assisted CT guidance not only facilitated the puncture procedure, but also significantly improved the efficacy. All 38 patients with 38 templates were accurately aligned, closely fitted to the skin marked lines with stable needles. Although the needles angles and depth in pre-operative plan were all deviated compared with intra-operative, the $\mathrm{D}_{90}, \mathrm{D}_{100}, \mathrm{~V}_{100}, \mathrm{~V}_{150}$, and $V_{200}$ showed no differences between pre-operative plan and post-operative plan. The reason for needles direction and depth deviation could be due to muscles relaxation after spinal anesthesia and OARs, such as blood, nerve, or colorectum motion during intra-operation as well as the needles pathway was very long for the pelvic recurrent location and the bony structure interference. The CI and HI did not show significant difference under intra-operative plan optimization, and both reached the pre-operative plan requirements.

3D-PNCT-assisted CT guidance ${ }^{125} \mathrm{I}-\mathrm{SAB}$ is an alternative option for cervical cancer patients with non-central pelvic recurrences. The care for cervical cancer patients with non-central pelvic recurrences is surgery or EBRT. To the best of our knowledge, there are few evidences to support the safety and effectiveness of ${ }^{125} \mathrm{I}-\mathrm{SAB}$ as an alternative to surgery or EBRT. Our study showed that, 
compared with surgery or EBRT for non-central pelvic recurrences in cervical cancer patients, ${ }^{125} \mathrm{I}$-SAB proved to be minimally invasive, effective, and with lower toxicity. It is an optimal salvage treatment for patients with non-central pelvic recurrent cervical cancer after EBRT.

In conclusion, 3D-PNCT-assisted CT guidance ${ }^{125}$ I-SAB demonstrated to be a feasible, safe, and accurate modality as a salvage therapy. It is also suitable for the aged patients or as a palliative therapy.

\section{Acknowledgments}

The authors would like to thank for the fund support from the National Key R\&D Program of China and National Natural and Science Foundation of China.

\section{Funding}

This work was supported by the National Key R\&D Program of China (2019YFB1311300 to J. Wang) and the National Natural Science Foundation of China (61631001 to J. Wang).

\section{Ethics approval and consent to participate}

All patients provided a written informed consent to participate in this study.

\section{Disclosure}

The authors report no conflict of interest.

\section{References}

1. Bray F, Ferlay J, Soerjomataram I et al. Global cancer statistics 2018: GLOBOCAN estimates of incidence and mortality worldwide for 36 cancers in 185 countries. CA Cancer J Clin 2018; 68: 394-424.

2. Quinn MA, Benedet JL, Odicino F et al. Carcinoma of the cervix uteri. FIGO 26th annual report on the results of treatment in gynecological cancer. Int J Gynaecol Obstet 2006; 95 Suppl 1: S43-103.

3. Pfaendler KS, Tewari KS. Changing paradigms in the systemic treatment of advanced cervical cancer. Am J Obstet Gynecol 2016; 214: 22-30.

4. Peiretti M, Zapardiel I, Zanagnolo V et al. Management of recurrent cervical cancer: a review of the literature. Surg Oncol 2012; 21: 59-66.

5. Legge F, Chiantera V, Macchia G et al. Clinical outcome of recurrent locally advanced cervical cancer (LACC) submitted to primary multimodality therapies. Gynecol Oncol 2015; 138: 83-88.

6. Perez CA, Grigsby PW, Camel HM et al. Irradiation alone or combined with surgery in stage IB, IIB carcinoma of uterine cervix: update of a nonrandomized comparison. Int J Radiat Oncol Biol Phys 1995; 31: 703-716.

7. Dornhöfer N, Höckel M. New developments in the surgical therapy of cervical carcinoma. Ann N Y Acad Sci 2008; 1138: 233-252.

8. Boussios S, Seraj E, Zarkavelis G et al. Management of patients with recurrent/advanced cervical cancer beyond first line platinum regimens: Where do we stand? A literature review. Crit Rev Oncol Hematol 2016; 108: 164-174.

9. Barney BM, Petersen IA, Dowdy SC et al. Intraoperative electron beam radiotherapy (IOERT) in the management of locally advanced or recurrent cervical cancer. Radiat Oncol 2013; 8: 80.

10. Kim YJ, Munsell MF, Park JC et al. Retrospective review of symptoms and palliative care interventions in women with advanced cervical cancer. Gynecol Oncol 2015; 139: 553-558.

11. Monk BJ, Lopez LM, Zarba JJ et al. Phase II, open-label study of pazopanib or lapatinib monotherapy combined with pazopanib plus lapatinib combination therapy in patients with advanced and recurrent cervical cancer. J Clin Oncol 2010; 28: 3562-3569.

12. Moore DH, Blessing JA, McQuellon RP et al. Phase III study of cisplatin with or without paclitaxel in stage IVB, recurrent, or persistent squamous cell carcinoma of the cervix: a gynecologic oncology group study. J Clin Oncol 2004; 22: 3113-3119.

13. Davis BJ, Horwitz EM, Lee WR et al. American Brachytherapy Society consensus guidelines for transrectal ultrasound-guided permanent prostate brachytherapy. Brachytherapy 2012; 11: 6-19.

14. Nag S, Beyer D, Friedland J et al. American Brachytherapy Society (ABS) recommendations for transperineal permanent brachytherapy of prostate cancer. Int J Radiat Oncol Biol Phys 1999; 44: 789-799.

15. Ji Z, Jiang $Y$, Tian $S$ et al. The effectiveness and prognostic factors of CT-Guided fadioactive I-125 seed implantation for the treatment of recurrent head and neck cancer after external beam radiation therapy. Int J Radiat Oncol Biol Phys 2019; 103: 638-645.

16. Huo X, Huo B, Wang $\mathrm{H}$ et al. Percutaneous computed tomography-guided permanent $125 \mathrm{I}$ implantation as therapy for pulmonary metastasis. J Contemp Brachytherapy 2018; 10 : 132-141.

17. Mo Z, Zhang T, Zhang Y et al. Feasibility and clinical value of CT-guided 125I brachytherapy for metastatic soft tissue sarcoma after first- line chemotherapy failure. Eur Radiol 2018; 28: 1194-1203.

18. Wang JJ, Yuan HS, Li JN et al. CT-guided radioactive seed implantation for recurrent rectal carcinoma after multiple therapy. Med Oncol 2010; 27: 421-429.

19. Huang MW, Zhang JG, Zheng L et al. Accuracy evaluation of a 3D-printed individual template for needle guidance in head and neck brachytherapy. J Radiat Res 2016; 57: 662-667.

20. Ji Z, Jiang Y, Guo F et al. Dosimetry verification of radioactive seed implantation for malignant tumors assisted by $3 \mathrm{D}$ printing individual templates and CT guidance. Appl Radiat Isot 2017; 124: 68-74.

21. Ji Z, Jiang Y, Su L et al. Dosimetry verification of 125I seeds implantation with three-dimensional printing noncoplanar templates and CT guidance for paravertebral/retroperitoneal malignant tumors. Technol Cancer Res Treat 2017; 16: 1044-1050.

22. Wang J, Zhang F, Guo J et al. Expert consensus workshop report: Guideline for three-dimensional printing template-assisted computed tomography-guided $125 \mathrm{I}$ seeds interstitial implantation brachytherapy. J Cancer Res Ther 2017; 13: 607-612.

23. van't Riet A, Mak AC, Moerland MA et al. A conformation number to quantify the degree of conformality in brachytherapy and external beam irradiation: application to the prostate. Int J Radiat Oncol Biol Phys 1997; 37: 731-736.

24. Saw CB, Suntharalingam N. Quantitative assessment of interstitial implants. Int J Radiat Oncol Biol Phys 1991; 20: 135-139.

25. Common Terminology Criteria for Adverse Events (CTCAE) version 5.0, https://evs.nci.nih.gov/ftp1/CTCAE/About. html (2017).

26. Westin SN, Rallapalli V, Fellman B et al. Overall survival after pelvic exenteration for gynecologic malignancy. Gynecol Oncol 2014; 134: 546-551. 
27. Petruzziello A, Kondo W, Hatschback SB et al. Surgical results of pelvic exenteration in the treatment of gynecologic cancer. World J Surg Oncol 2014; 12: 279.

28. Sardain H, Lavoue V, Redpath M et al. Curative pelvic exenteration for recurrent cervical carcinoma in the era of concurrent chemotherapy and radiation therapy. A systematic review. Eur J Surg Oncol 2015; 41: 975-985.

29. Jiang YL, Meng N, Wang JJ et al. Percutaneous computed tomography/ultrasonography-guided permanent iodine-125 implantation as salvage therapy for recurrent squamous cell cancers of head and neck. Cancer Biol Ther 2010; 9: 959-966.

30. Ji Z, Jiang Y, Guo F et al. Radiation-related adverse effects of CT-guided implantation of $125 \mathrm{I}$ seeds for thoracic recurrent and/or metastatic malignancy. Sci Rep 2019; 9: 14803.

31. Yao L, Cao Q, Wang J et al. CT-guided 125I seed interstitial brachytherapy as a salvage treatment for recurrent spinal metastases after external beam radiotherapy. Biomed Res Int 2016; 2016: 8265907.

32. Mo Z, Zhang T, Zhang Y et al. Feasibility and clinical value of CT-guided 125I brachytherapy for metastatic soft tissue sarcoma after first-line chemotherapy failure. Eur Radiol 2018; 28: 1194-1203.

33. Zhang FJ, Li CX, Zhang L et al. Short-to mid-term evaluation of CT guided 125I brachytherapy on intra-hepatic recurrent tumors and/or extra-hepatic metastases after liver transplantation for hepatocellular carcinoma. Cancer Biol Ther 2009; 8: 585-590.

34. Qu A, Jiang P, Sun H et al. Efficacy and dosimetry analysis of image-guided radioactive $125 \mathrm{I}$ seed implantation as salvage treatment for pelvic recurrent cervical cancer after external beam radiotherapy. J Gynecol Oncol 2019; 30: e9.

35. VanKoevering KK, Hollister SJ, Green GE. Advanced in 3-dimensional printing in otolaryngology. JAMA Ontolaryngol 2017; 143: 178-183.

36. Chen MY, Skewes J, Desselle M et al. Current applications of three-dimensional printing in urology. BJU Int 2020; 125: $17-27$.

37. Gross BC, Erkal JL, Lockwood SY et al. Evaluation of 3D printing and its potential impact on biotechnology and the chemical sciences. Anal Chem 2014; 86: 3240-3253.

38. Jiang Y, Ji Z, Guo F et al. Side effects of CT-guided implantation of 125I seeds for recurrent malignant tumors of the head and neck assisted by 3D printing non co-planar template. Radiat Oncol 2018; 13: 18.

39. Ji Z, Jiang Y, Guo F et al. Safety and efficacy of CT-guided radioactive iodine-125 seed implantation assisted by a $3 \mathrm{D}$ printing template for the treatment of thoracic malignancies. J Cancer Res Clin Oncol 2020; 146: 229-236.

40. Ji Z, Sun H, Jiang Y et al. Comparative study for CT-guided 125I seed implantation assisted by 3D printing coplanar and non-coplanar template in peripheral lung cancer. J Contemp Brachytherapy 2019; 11: 169-173.

41. Liu S, Wang H, Wang C et al. Dosimetry verification of 3D-printed individual template based on CT-MRI fusion for radioactive 125I seed implantation in recurrent high-grade gliomas. J Contemp Brachytherapy 2019; 11: 235-242. 